\title{
High Grade Sarcoma
}

National Cancer Institute

\section{Source}

National Cancer Institute. High Grade Sarcoma. NCI Thesaurus. Code C9418.

A sarcoma with the morphologic features of a high grade tumor and lack of differentiation. 